\title{
HOMOLOGICAL LOCAL LINKING
}

\author{
KANISHKA PERERA
}

\begin{abstract}
We generalize the notion of local linking to include certain cases where the functional does not have a local splitting near the origin. Applications to second-order Hamiltonian systems are given.
\end{abstract}

\section{INTRODUCTION}

The notion of local linking introduced by Li and Liu [4] plays a useful role in a wide variety of problems in the Calculus of Variations. Let $F$ be a real $C^{1}$ function defined on a Banach space $X$. We say that $F$ has a local linking near the origin if $X$ has a direct sum decomposition $X=X_{1} \oplus X_{2}$ with $j=\operatorname{dim} X_{1}<\infty, F(0)=0$, and, for some $r>0$,

$$
\left\{\begin{array}{l}
F(u) \leq 0 \text { for } u \in X_{1},\|u\| \leq r, \\
F(u)>0 \text { for } u \in X_{2}, 0<\|u\| \leq r .
\end{array}\right.
$$

Then it is clear that 0 is a critical point of $F$.

In this paper we give the following more general definition of "homological" local linking:

Definition 1.1. Assume that 0 is an isolated critical point of $F$ with $F(0)=$ 0 and let $q, \beta$ be positive integers. We say that $F$ has a local $(q, \beta)$-linking near the origin if there exist a neighborhood $U$ of 0 and subsets $A, S, B$ of $U$ with $A \cap S=\emptyset, 0 \notin A, A \subset B$ such that

1. 0 is the only critical point of $F$ in $F_{0} \cap U$ where $F_{0}$ is the sublevel set $\{u \in X: F(u) \leq 0\}$,

2. denoting by $i_{1 *}: H_{q-1}(A) \longrightarrow H_{q-1}(U \backslash S)$ and $i_{2 *}: H_{q-1}(A) \longrightarrow$ $H_{q-1}(B)$ the embeddings of the singular homology groups induced by inclusions,

$$
\operatorname{rank} i_{1 *}-\operatorname{rank} i_{2 *} \geq \beta,
$$

1991 Mathematics Subject Classification. Primary: 58E05.

Key words and phrases. Morse theory, critical groups, local linking.

Received: March 10, 1998. 
3. $F \leq 0$ on $B$,

4. $F>0$ on $S \backslash\{0\}$.

If $F$ satisfies the condition (1) with $j \geq 1$ and 0 is an isolated critical point of $F$, taking $U$ to be a sufficiently small closed ball $B_{\rho}$ centered at the origin, $A=\partial B_{\rho} \cap X_{1}, S=B_{\rho} \cap X_{2}$, and $B=B_{\rho} \cap X_{1}$, we see that $F$ has a local $(j, 1)$-linking near the origin. The following example in $\mathbb{R}^{\not \models}$ shows that our definition is, in fact, weaker than (1):

Example 1.2 (Monkey saddle). $F(x, y)=x^{3}-3 x y^{2}$ has a local $(1,2)$ linking near the origin; see Proposition 2.1.

Note that the critical groups of $F$ at 0 are given by

$$
C_{*}(F, 0)=H_{*}\left(F_{0} \cap U,\left(F_{0} \cap U\right) \backslash\{0\}\right)
$$

(see Chang [2] or Mawhin and Willem [8]). It was proved in Liu [7] that if $F$ satisfies (1) and 0 is an isolated critical point of $F$, then $C_{j}(F, 0) \neq 0$. This fact was used in Perera [9] to obtain a nontrivial critical point $u$ with either $C_{j+1}(F, u) \neq 0$ or $C_{j-1}(F, u) \neq 0$, under an additional assumption on the behavior of $F$ at infinity. Here we extend these results to the case where $F$ satisfies the weaker conditions given in Definition 1.1 near the origin. As an application we prove the existence of nontrivial time-periodic solutions of a system of ordinary differential equations, under different hypotheses on the behavior of the nonlinearity at infinity.

For the existence of nontrivial critical points under the usual definition of local linking and various assumptions at infinity see $\mathrm{Li}$ and $\mathrm{Liu}$ [4], $\mathrm{Li}$ and Liu [5], Liu [7], Silva [10], Brézis and Nirenberg [1], Li and Willem [6], and their references.

\section{An ExAmple}

As an example of homological local linking, we prove the following proposition:

Proposition 2.1. Assume that 0 is an isolated critical point of $F$ and

$$
F(u)=P(u)+o\left(\|u\|^{s}\right) \text { at } u=0
$$

where $P$ is homogeneous of degree $s>1$, i.e.,

$$
P(\lambda u)=\lambda^{s} P(u) \quad \forall \lambda \geq 0, \forall u \in X .
$$

Assume also that there are disjoint subsets $\tilde{A}, \tilde{S}$ of $S^{\infty}$, the unit sphere in $X$, such that

1. the rank of the embedding $H_{q-1}(\tilde{A}) \longrightarrow H_{q-1}\left(S^{\infty} \backslash \tilde{S}\right)$ is at least $\beta+\delta_{q 1}$,

2. $\sup _{\tilde{A}} P<0$,

3. $\inf _{\tilde{S}} P>0$.

Then $F$ has a local $(q, \beta)$-linking near the origin. 
Proof. Fix $\epsilon>0$ so that $\sup _{\tilde{A}} P+\epsilon<0<\inf _{\tilde{S}} P-\epsilon$ and take $\rho>0$ sufficiently small such that 0 is the only critical point of $F$ in $U=B_{\rho}=$ $\{u \in X:\|u\| \leq \rho\}$ and

$$
|F(u)-P(u)| \leq \epsilon\|u\|^{s} \text { for }\|u\| \leq \rho .
$$

Then take

$$
\begin{aligned}
A & =\{\rho u: u \in \tilde{A}\} \\
B & =\{\lambda u: u \in \tilde{A}, 0 \leq \lambda \leq \rho\}, \\
S & =\{\lambda u: u \in \tilde{S}, 0 \leq \lambda \leq \rho\} .
\end{aligned}
$$

On $B$,

$$
F(\lambda u) \leq P(\lambda u)+\epsilon\|\lambda u\|^{s}=\lambda^{s}(P(u)+\epsilon) \leq \lambda^{s}\left(\sup _{\tilde{A}} P+\epsilon\right) \leq 0
$$

and on $S \backslash\{0\}$,

$$
F(\lambda u) \geq P(\lambda u)-\epsilon\|\lambda u\|^{s}=\lambda^{s}(P(u)-\epsilon) \geq \lambda^{s}\left(\inf _{\tilde{S}} P-\epsilon\right)>0 .
$$

Now we verify the condition 2 of Definition 1.1. Set $S_{\rho}=\partial B_{\rho}$ and $\tilde{S}_{\rho}=\{\rho u: u \in \tilde{S}\}$. By the assumption 1, the rank of the embedding $i_{*}: H_{q-1}(A) \longrightarrow H_{q-1}\left(S_{\rho} \backslash \tilde{S}_{\rho}\right)$ is at least $\beta+\delta_{q 1}$. The map

$$
(t, \lambda u) \longmapsto[(1-t) \lambda+t] u \quad 0 \leq t \leq 1, u \in S^{\infty} \backslash \tilde{S}, 0<\lambda \leq \rho
$$

is a strong deformation retraction of $B_{\rho} \backslash S$ onto $S_{\rho} \backslash \tilde{S}_{\rho}$ and hence the embedding $H_{q-1}\left(S_{\rho} \backslash \tilde{S}_{\rho}\right) \longrightarrow H_{q-1}\left(B_{\rho} \backslash S\right)$ is an isomorphism. It follows that

$$
\operatorname{rank} i_{1 *}=\operatorname{rank} i_{*} \geq \beta+\delta_{q 1} .
$$

On the other hand, $B$ is contractible to 0 via

$$
(t, \lambda u) \longmapsto(1-t) \lambda u \quad 0 \leq t \leq 1, u \in \tilde{A}, 0 \leq \lambda \leq \rho
$$

and hence

$$
\operatorname{rank} i_{2 *}=\delta_{q 1}
$$

\section{Critical Groups of the Origin and \\ Nontrivial Critical Points}

The following theorem extends Theorem 2.1 of Liu [7]:

Theorem 3.1. If $F$ has a local $(q, \beta)$-linking near the origin, then

$$
\operatorname{rank} C_{q}(F, 0) \geq \beta \text {. }
$$


Proof. Consider the following portion of the exact sequence of the pair $\left(F_{0} \cap\right.$ $\left.U, F_{0} \cap U \backslash\{0\}\right)$ :

$$
C_{q}(F, 0) \stackrel{\partial_{*}}{\longrightarrow} H_{q-1}\left(F_{0} \cap U \backslash\{0\}\right) \stackrel{i_{*}}{\longrightarrow} H_{q-1}\left(F_{0} \cap U\right)
$$

We have

$$
\operatorname{rank} C_{q}(F, 0) \geq \operatorname{rank} \partial_{*}=\text { nullity } i_{*} .
$$

Consider the following commutative diagram induced by inclusions:

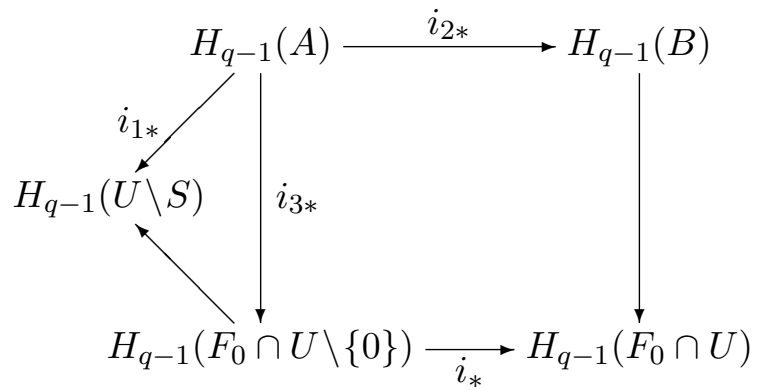

We have

$$
\begin{aligned}
\operatorname{nullity} i_{*} & \geq \operatorname{rank} i_{3 *}-\operatorname{rank} i_{2 *} \\
& \geq \operatorname{rank} i_{1 *}-\operatorname{rank} i_{2 *} \\
& \geq \beta .
\end{aligned}
$$

Now we assume that $F$ satisfies the Palais-Smale compactness condition (PS) and has only isolated critical values, with each critical value corresponding to a finite number of critical points, and set

$$
\begin{aligned}
& K_{c}=\text { set of critical points of } F \text { where } F=c, \\
& K_{a}^{b}=\text { set of critical points of } F \text { where } a<F<b .
\end{aligned}
$$

The main result of this section is the following:

Theorem 3.2. Suppose that $F$ has a local $(q, \beta)$-linking near the origin and assume that there are regular values $a, b$ of $F$ such that $a<0<b$ and

$$
\operatorname{rank} H_{q}\left(F_{b}, F_{a}\right)<\beta \text {. }
$$

Then

$$
\sum_{u \in K_{a}^{0}} \operatorname{rank} C_{q-1}(F, u)+\sum_{u \in K_{0}^{b}} \operatorname{rank} C_{q+1}(F, u) \geq \beta-\operatorname{rank} H_{q}\left(F_{b}, F_{a}\right) .
$$

In particular, $F$ has a (nontrivial) critical point $u$ with either

$$
\begin{gathered}
a<F(u)<0 \text { and } C_{q-1}(F, u) \neq 0, \text { or } \\
0<F(u)<b \text { and } C_{q+1}(F, u) \neq 0 .
\end{gathered}
$$

Theorem 3.2 follows from Lemma 3.3 below and Theorem 3.1. 
Lemma 3.3. If $a<b$ are regular values of $F, c \in(a, b)$, and $q \in \mathbb{Z}$, then

$$
\begin{aligned}
& \sum_{u \in K_{a}^{c}} \operatorname{rank} C_{q-1}(F, u)+\sum_{u \in K_{c}^{b}} \operatorname{rank} C_{q+1}(F, u) \\
\geq & \sum_{u \in K_{c}} \operatorname{rank} C_{q}(F, u)-\operatorname{rank} H_{q}\left(F_{b}, F_{a}\right) .
\end{aligned}
$$

The proof of Lemma 3.3 makes use of the following topological lemma:

Lemma 3.4. If $B^{\prime} \subset B \subset A \subset A^{\prime}$ are topological spaces and $q \in \mathbb{Z}$, then

$$
\begin{aligned}
& \operatorname{rank} H_{q-1}\left(B, B^{\prime}\right)+\operatorname{rank} H_{q+1}\left(A^{\prime}, A\right) \\
\geq & \operatorname{rank} H_{q}(A, B)-\operatorname{rank} H_{q}\left(A^{\prime}, B^{\prime}\right) .
\end{aligned}
$$

Proof. Consider the following portions of the exact sequences of the triples $\left(A, B, B^{\prime}\right)$ and $\left(A^{\prime}, A, B^{\prime}\right)$, respectively:

$$
\begin{gathered}
H_{q}\left(A, B^{\prime}\right) \stackrel{j_{*}}{\longrightarrow} H_{q}(A, B) \stackrel{\partial_{*}}{\longrightarrow} H_{q-1}\left(B, B^{\prime}\right), \\
H_{q+1}\left(A^{\prime}, A\right) \stackrel{\partial_{*}}{\longrightarrow} H_{q}\left(A, B^{\prime}\right) \stackrel{i_{*}}{\longrightarrow} H_{q}\left(A^{\prime}, B^{\prime}\right)
\end{gathered}
$$

We have

$$
\begin{aligned}
\operatorname{rank} H_{q-1}\left(B, B^{\prime}\right)+\operatorname{rank} H_{q}\left(A, B^{\prime}\right) & \geq \operatorname{rank} H_{q}(A, B), \\
\operatorname{rank} H_{q}\left(A^{\prime}, B^{\prime}\right)+\operatorname{rank} H_{q+1}\left(A^{\prime}, A\right) & \geq \operatorname{rank} H_{q}\left(A, B^{\prime}\right) .
\end{aligned}
$$

Proof of Lemma 3.3. Take $\epsilon>0$ such that $a<c-\epsilon<c+\epsilon<b$ and $c$ is the only critical value of $F$ in $[c-\epsilon, c+\epsilon]$. Applying Lemma 3.4 to $F_{a} \subset F_{c-\epsilon} \subset F_{c+\epsilon} \subset F_{b}$,

$$
\begin{aligned}
& \operatorname{rank} H_{q-1}\left(F_{c-\epsilon}, F_{a}\right)+\operatorname{rank} H_{q+1}\left(F_{b}, F_{c+\epsilon}\right) \\
\geq & \operatorname{rank} H_{q}\left(F_{c+\epsilon}, F_{c-\epsilon}\right)-\operatorname{rank} H_{q}\left(F_{b}, F_{a}\right) .
\end{aligned}
$$

But, by Chapter I, Theorem 4.3, Corollary 4.1, and Theorem 4.2 of Chang $[2]$,

$$
\begin{aligned}
\operatorname{rank} H_{q-1}\left(F_{c-\epsilon}, F_{a}\right) & \leq \sum_{u \in K_{a}^{c}} \operatorname{rank} C_{q-1}(F, u) \\
\operatorname{rank} H_{q+1}\left(F_{b}, F_{c+\epsilon}\right) & \leq \sum_{u \in K_{c}^{b}} \operatorname{rank} C_{q+1}(F, u) \\
\operatorname{rank} H_{q}\left(F_{c+\epsilon}, F_{c-\epsilon}\right) & =\sum_{u \in K_{c}} \operatorname{rank} C_{q}(F, u) .
\end{aligned}
$$

The following corollary generalizes Theorem 2.2 of Liu [7] and Theorem 5 of Brézis and Nirenberg [1]. See Remark 2.3 of Liu [7] and the remarks following Theorem 4 and proof of Theorem 5 of Brézis and Nirenberg [1] for the history of this result: 
Corollary 3.5 (Three Critical Point Theorem). Suppose that F has a local $(q, \beta)$-linking near the origin and assume that $F$ is bounded below. If $q=1$ and $\beta \geq 2$, or $q \geq 2$, then $F$ has at least two nontrivial critical points. If $q \geq 2$, then $F$ has a nontrivial critical point which is not a local minimizer.

Proof. $F$ achieves its minimum at some point $u_{0}$ with

$$
F\left(u_{0}\right) \leq 0 \text { and } \operatorname{rank} C_{j}\left(F, u_{0}\right)=\delta_{j 0}
$$

(for the critical groups of an isolated local minimum point see Example 1 in Chapter I, Section 4 of Chang [2]). By Theorem 3.1,

$$
C_{q}(F, 0) \neq 0
$$

and hence $u_{0} \neq 0$. Supposing 0 and $u_{0}$ to be the only critical points and taking $a<\inf _{X} F$ and $b=+\infty$ in Theorem 3.2, we have

$$
\beta \leq \delta_{q 1}
$$

If $q \geq 2$, the critical point $u \neq 0$ with either

$$
C_{q-1}(F, u) \neq 0 \text { or } C_{q+1}(F, u) \neq 0
$$

obtained in Theorem 3.2 is not a local minimizer.

\section{SeCOnd-Order Hamiltonian systems}

Consider the second-order nonautonomous system

$$
\ddot{x}=\nabla V(t, x)
$$

where $V \in C^{1}\left(\mathbb{R} \times \mathbb{R}^{\ltimes}, \mathbb{R}\right)$ is $2 \pi$-periodic in $t$ and satisfies

$\left(V_{1}\right)$ : there are constants $\mu>2$ and $R>0$ such that

$$
0<\mu V(t, x) \leq x \cdot \nabla V(t, x) \text { for }|x| \geq R, \forall t,
$$

$\left(V_{2}\right)$ : there is a homogeneous function $P \in C^{1}\left(\mathbb{R}^{\ltimes}, \mathbb{R}\right)$ of degree $s>2$ such that

$$
V(t, x)=P(x)+o\left(|x|^{s}\right) \text { at } x=0 \text {, uniformly in } t .
$$

Theorem 4.1. Assume $\left(V_{1}\right),\left(V_{2}\right)$, and the following condition on $P$ :

$(P)$ : there are disjoint subsets $\tilde{A}, \tilde{S}$ of $S^{n-1}$ such that

1. for some positive integers $q \leq n$ and $\beta$, the rank of the embedding $H_{q-1}(\tilde{A}) \longrightarrow H_{q-1}\left(S^{n-1} \backslash \tilde{S}\right)$ is at least $\beta+\delta_{q 1}$,

2. $P<0$ on $\tilde{A}$,

3. $P>0$ on $\tilde{S}$.

Then (2) has at least one nonzero $2 \pi$-periodic solution.

Remark 4.2. Our assumption $(P)$ generalizes the condition $\left(P_{4}\right)$ of Felmer and Silva [3]. See Theorem 7 and the remark following it in Li and Willem [6] for related results. 
Proof of Theorem 4.1. We seek solutions of (2) as critical points of the functional

$$
F(x)=\int_{0}^{2 \pi}\left[\frac{1}{2}|\dot{x}|^{2}+V(t, x(t))\right] d t
$$

defined on the Hilbert space $X$ of vector functions $x(t)$ having period $2 \pi$ and belonging to $H^{1}$ on $[0,2 \pi]$, with the norm

$$
\|x\|=\frac{1}{\sqrt{2 \pi}}\left[\int_{0}^{2 \pi}|\dot{x}|^{2}+|x|^{2}\right]^{1 / 2} .
$$

It is well-known that $F$ satisfies (PS). As in the proof of Lemma 3.2 of Wang [11], $\left(V_{1}\right)$ also implies that

$$
H_{j}\left(X, F_{a}\right)=0 \quad \forall j \in \mathbb{Z}
$$

for $a<0$ and $|a|$ sufficiently large. The conclusion follows from Theorem 3.2 and the Lemma 4.3 below.

Lemma 4.3. If $V$ satisfies $\left(V_{2}\right)$ with $P$ as in Theorem 4.1, then $F$ has a local $(q, \beta)$-linking near the origin.

Proof. We have the splitting $X=X_{1} \oplus X_{2}$ where $X_{1}$ is the space of constant functions, identified with $\mathbb{R}^{\ltimes}$, and $X_{2}$ is the space of functions in $X$ whose integral is zero. We take

$$
\begin{aligned}
U & =\left\{\lambda x_{1}+x_{2}: x_{1} \in S^{n-1}, x_{2} \in X_{2}, 0 \leq \lambda \leq \rho,\left\|x_{2}\right\| \leq \rho\right\}, \\
A & =\left\{\rho x_{1}: x_{1} \in \tilde{A}\right\} \\
B & =\left\{\lambda x_{1}: x_{1} \in \tilde{A}, 0 \leq \lambda \leq \rho\right\}, \\
S & =\left\{\lambda x_{1}+x_{2}: x_{1} \in \tilde{S}, x_{2} \in X_{2}, 0 \leq \lambda \leq \rho,\left\|x_{2}\right\| \leq \rho\right\} .
\end{aligned}
$$

As in the proof of Proposition 2.1, $F \leq 0$ on $B$ for $\rho>0$ sufficiently small. On $S \backslash\{0\}$,

$$
F\left(\lambda x_{1}+x_{2}\right)=\int \frac{1}{2}\left|\dot{x}_{2}\right|^{2}+P\left(\lambda x_{1}+x_{2}\right)+o(1)\left|\lambda x_{1}+x_{2}\right|^{s} \text { as } \rho \rightarrow 0 .
$$

By the mean value theorem and the Young's inequality,

$$
\begin{aligned}
P\left(\lambda x_{1}+x_{2}\right) & =P\left(\lambda x_{1}\right)+\nabla P\left(\lambda x_{1}+\theta x_{2}\right) \cdot x_{2} \text { for some } \theta \in[0,1] \\
& \geq \lambda^{s} P\left(x_{1}\right)-C\left|\lambda x_{1}+\theta x_{2}\right|^{s-1}\left|x_{2}\right| \\
& \geq \lambda^{s} \min _{\tilde{S}} P-C\left(\lambda^{(s-1) p}+\left|x_{2}\right|^{p^{\prime}}+\left|x_{2}\right|^{s}\right)
\end{aligned}
$$

where $p, p^{\prime}$ are conjugate exponents with $2<p^{\prime}<s$. It follows that

$$
\begin{aligned}
F\left(\lambda x_{1}+x_{2}\right) \geq & \lambda^{s}\left(2 \pi \min _{\tilde{S}} P-C \rho^{\left(s / p^{\prime}-1\right) p}+o(1)\right) \\
& +\int \frac{1}{2}\left|\dot{x}_{2}\right|^{2}-C\left(\left\|x_{2}\right\|^{p^{\prime}}+\left\|x_{2}\right\|^{s}\right)>0
\end{aligned}
$$

on $S$ for small $\rho$. 
Set $U_{1}=\left\{x_{1} \in X_{1}:\left|x_{1}\right| \leq \rho\right\}$ and $S_{1}=\left\{\lambda x_{1}: x_{1} \in \tilde{S}, 0 \leq \lambda \leq \rho\right\}$. As in the proof of Proposition 2.1, the rank of the embedding $H_{q-1}(A) \longrightarrow$ $H_{q-1}\left(U_{1} \backslash S_{1}\right)$ is at least $\beta+\delta_{q 1}$. The map

$$
\begin{aligned}
\left(t, \lambda x_{1}+x_{2}\right) \longmapsto \lambda x_{1}+(1-t) x_{2} \quad & 0 \leq t \leq 1, x_{1} \in S^{n-1} \backslash \tilde{S}, x_{2} \in X_{2}, \\
& 0<\lambda \leq \rho,\left\|x_{2}\right\| \leq \rho
\end{aligned}
$$

is a strong deformation retraction of $U \backslash S$ onto $U_{1} \backslash S_{1}$, and hence the embedding $H_{q-1}\left(U_{1} \backslash S_{1}\right) \longrightarrow H_{q-1}(U \backslash S)$ is an isomorphism.

Remark 4.4. Note that Theorem 3.2 gives a critical point $x$ with either

$$
\begin{gathered}
F(x)<0 \text { and } C_{q-1}(F, x) \neq 0, \text { or } \\
F(x)>0 \text { and } C_{q+1}(F, x) \neq 0,
\end{gathered}
$$

yielding Morse index estimates for $x$ via the Shifting theorem when $V$, and hence $F$, is $C^{2}$ (see Chapter I, Theorem 5.4 of Chang [2]): either

$$
\begin{gathered}
F(x)<0 \text { and } m(x) \leq q-1 \leq m^{*}(x) \text {, or } \\
F(x)>0 \text { and } m(x) \leq q+1 \leq m^{*}(x)
\end{gathered}
$$

where $m(x)$ and $m^{*}(x)=m(x)+\operatorname{dim} \operatorname{ker} d^{2} F(x)$ denote the Morse index and the large Morse index of $x$, respectively. This additional information can sometimes be used to distinguish $x$ from the constant solutions, when they exist.

Now we replace $\left(V_{1}\right)$ by the condition

$\left(V_{1}\right)^{\prime}: V(t, x) \rightarrow+\infty$ as $|x| \rightarrow \infty$ uniformly in $t$,

which implies that $F$ satisfies (PS) and is bounded below. Then Corollary 3.5 yields

Theorem 4.5. Assume $\left(V_{1}\right)^{\prime},\left(V_{2}\right)$, and $(P)$. If $q=1$ and $\beta \geq 2$, or $q \geq 2$, then (2) has at least two nonzero $2 \pi$-periodic solutions.

Remark 4.6. See Theorems 7 and 7' in Brézis and Nirenberg [1] for related results.

\section{REFERENCES}

1. H. Brézis and L. Nirenberg, Remarks on finding critical points, Comm. Pure Appl. Math. XLIV (1991), 939-963.

2. K.-C. Chang, Infinite-dimensional Morse theory and multiple solution problems, Progress in Nonlinear Differential Equations and their Applications, \#6, Birkhäuser, Boston, 1993.

3. P. L. Felmer and E.-A. de B. e Silva, Subharmonics near an equilibrium for some second-order Hamiltonian systems, Proc. Roy. Soc. Edinburgh Sect. A, 123 (1993), 819-834.

4. S. J. Li and J. Q. Liu, Some existence theorems on multiple critical points and their applications, Kexue Tongbao, 17 (1984), 1025-1027.

5. __ Morse theory and asymptotically linear Hamiltonian systems, J. Differential Equations, 78 (1989), 53-73.

6. S. J. Li and M. Willem, Applications of local linking to critical point theory, J. Math. Anal. Appl. 189 (1995), 6-32.

7. J. Liu, The Morse index of a saddle point, Systems Sci. Math. Sci. 2 (1989), 32-39. 
8. J. Mawhin and M. Willem, Critical point theory and Hamiltonian systems, Applied Mathematical Sciences, \#74, Springer-Verlag, New York, 1989.

9. K. Perera, Critical groups of critical points produced by local linking with applications, preprint.

10. E.-A. de B. e Silva, Linking theorems and applications to semilinear elliptic problems at resonance, Nonlinear Anal. 16 (1991), 455-477.

11. Z. Q. Wang, On a superlinear elliptic equation, Ann. Inst. H. Poincaré Anal. Non Linéaire, 8 (1991), 43-58.

Department of Mathematics

University of California Irvine

IRvine, CA 92697-3875, USA

E-mail: kperera@math.uci.edu 


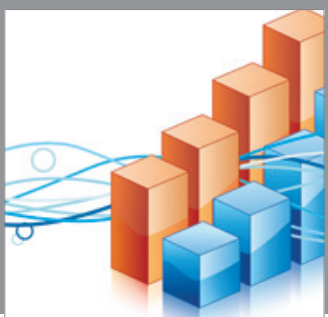

Advances in

Operations Research

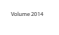

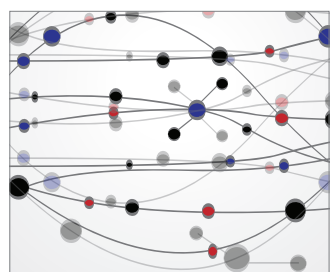

\section{The Scientific} World Journal
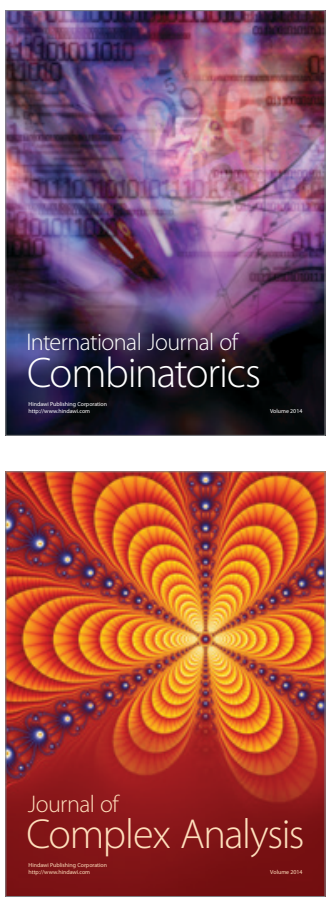

International Journal of

Mathematics and

Mathematical

Sciences
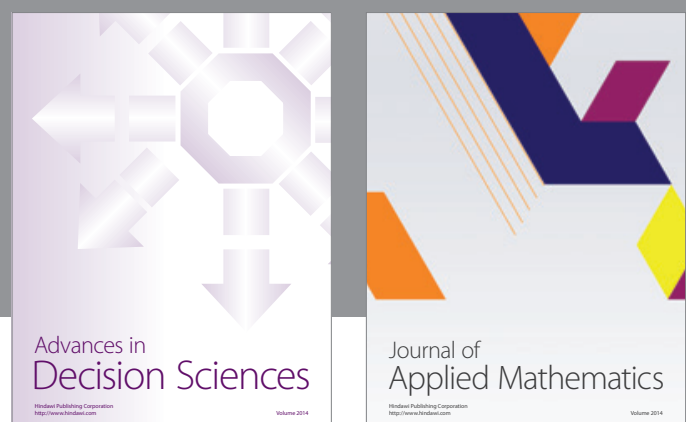

Journal of

Applied Mathematics
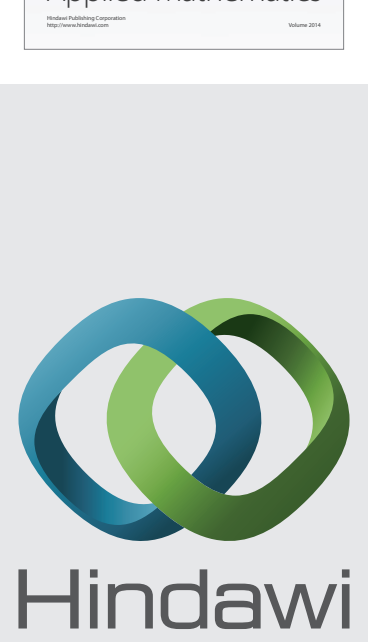

Submit your manuscripts at http://www.hindawi.com
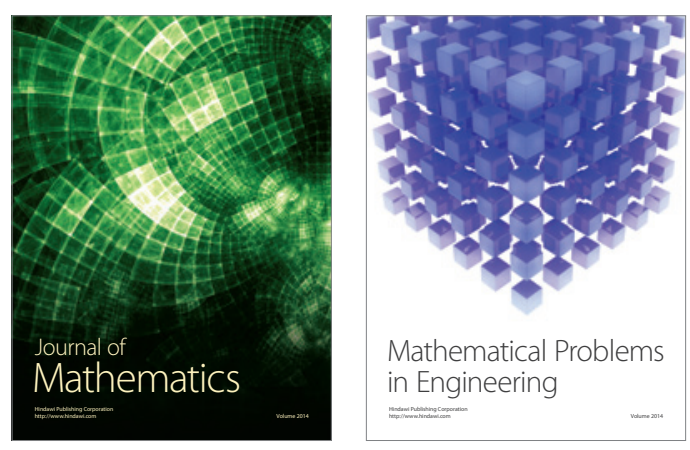

Mathematical Problems in Engineering
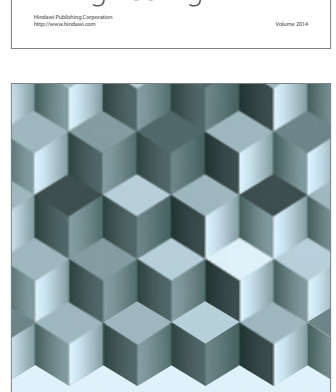

Journal of

Function Spaces
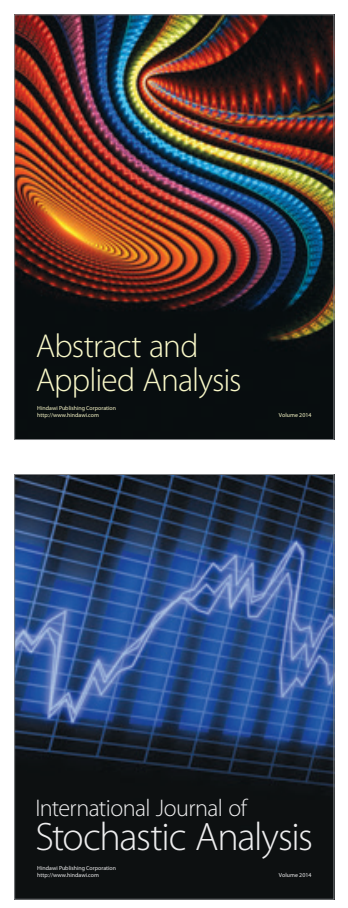

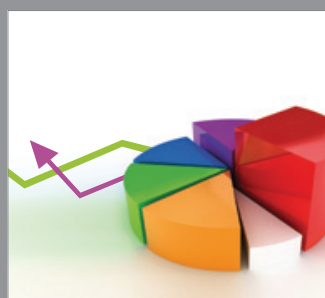

ournal of

Probability and Statistics

Promensencen
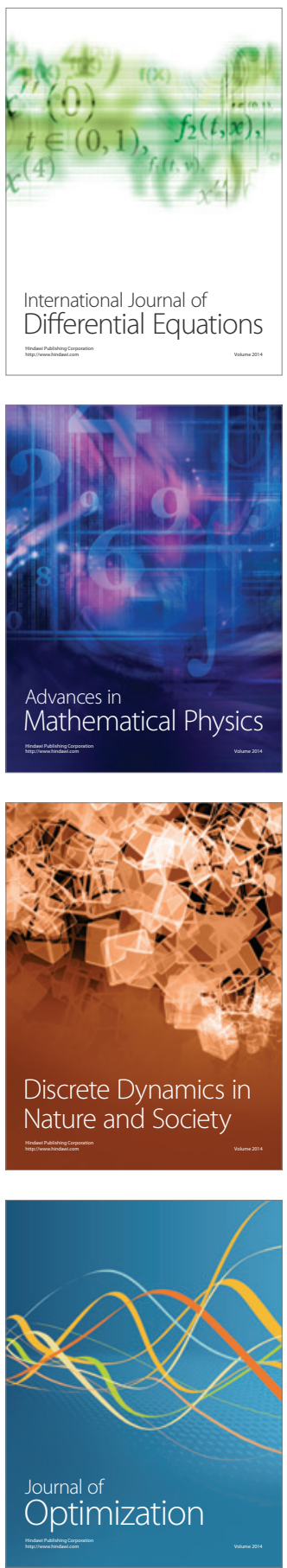